\title{
Complex derivatives valuation: applying the Least-Squares Monte Carlo Simulation Method with several polynomial basis
}

\author{
Ursula Silveira Monteiro de Lima* and Carlos Patricio Samanez
}

*Correspondence: ursula.
smonteiro@gmail.com
Department of Industrial
Engineering, Pontifical Catholic
University of Rio de Janeiro,
Marquês de São Vicente Street, 225
/ 22453-900 Gávea, Rio de Janeiro,
Brazil

* Correspondence: ursula. smonteiro@gmail.com Engineering, Pontifical Catholic University of Rio de Janeiro, / 22453-900 Gávea, Rio de Janeiro, Brazil

\begin{abstract}
Background: This article investigates the Least-Squares Monte Carlo Method by using different polynomial basis in American Asian Options pricing. The standard approach in the option pricing literature is to choose the basis arbitrarily. By comparing four different polynomial basis we show that the choice of basis interferes in the option's price.
\end{abstract}

Methods: We assess Least-Squares Method performance in pricing four different American Asian Options by using four polynomial basis: Power, Laguerre, Legendre and Hermite A. To every American Asian Option priced, three sets of parameters are used in order to evaluate it properly.

Results: We show that the choice of the basis interferes in the option's price by showing that one of them converges to the option's value faster than any other by using fewer simulated paths. In the case of an Amerasian call option, for example, we find that the preferable polynomial basis is Hermite A. For an Amerasian put option, the Power polynomial basis is recommended. Such empirical outcome is theoretically unpredictable, since in principle all basis can be indistinctly used when pricing the derivative.

Conclusion: In this article The Least-Squares Monte Carlo Method performance is assessed in pricing four different types of American Asian Options by using four different polynomial basis through three different sets of parameters. Our results suggest that one polynomial basis is best suited to perform the method when pricing an American Asian option. Theoretically all basis can be indistinctly used when pricing the derivative. However, our results does not confirm these. We find that when pricing an American Asian put option, Power $A$ is better than the other basis we have studied here whereas when pricing an American Asian call, Hermite A is better.

Keywords: Complex derivatives valuation, Least-Squares Monte Carlo Method, Amerasian options, Polynomial basis

\section{包 Springer}

(c) 2016 de Lima and Samanez. Open Access This article is distributed under the terms of the Creative Commons Attribution 4.0 International License (http://creativecommons.org/licenses/by/4.0/), which permits unrestricted use, distribution, and reproduction in any medium, provided you give appropriate credit to the original author(s) and the source, provide a link to the Creative Commons license, and indicate if changes were made. 


\section{Background}

Asian options are often used for cash flow hedges in companies whose purchase programming is set to mitigate the fluctuation of raw materials' prices. Its versatility is confirmed by its presence in markets like commodities, electric power, interest rates and currency rates (McDonald 2006). Because they are complex (or exotic), the Asian options are usually traded over the counter. The characteristics of the contract (subject, premium, strike price, deadlines and maturity) are freely agreed between the parties, emphasizing their non-standardization.

Traditional techniques such as the finite-differences method and lattice become less attractive when dealing with pricing derivatives with multiple stochastic variables, problems with many dimensions, or even path-dependent American options, as it is the case of American Asian (Amerasian) options. The most flexible technique for pricing exotic options, such as American options, is the use of stochastic simulation with optimization algorithm. This technique includes different methods, such as the Least-Squares Monte Carlo method (LSM), first introduced by Longstaff and Schwartz (2001). Besides being faster and more precise to compute than other methodologies, the LSM methodology helps assess path-dependent American options with multiple dimensions and multiple state variables, being also applied to Markovian and non-Markovian problems.

The Least-Squares Monte Carlo Method has been used to price American Asian options. Longstaff and Schwartz (2001) exemplified the use of their technique in pricing an American Asian Arithmetic Average Fixed Strike call option with a specific polynomial basis, i.e., power. Moreno and Navas (2003) access the performance of Least Squares Monte Carlo Method numerically, by using two different polynomial basis, i.e., Laguerre and Hermite B, to value the same American Asian option priced by Longstaff and Schwartz (2001). Moreno and Navas (2003) found that the choice of the polynomial basis and the degree of these polynomials influence the estimated prices. Chaudhary (2005) used quasi-random sequences to improve the performance of this technique by pricing an American Asian Arithmetic Average call option without varying the polynomial basis used. In their experiment, they used power polynomial basis. Also to access the performance of this techniques, Cerrato and Cheung (2007) priced an American Asian Arithmetic Average call option using three sets of simulated trajectories. They applied two different polynomial basis, i.e., exponential and Laguerre. Although not dedicated to the task of pricing an American Asian option, other authors, such as Glasserman (2004) states that "accuracy depends on the choice of basis functions, which may require experimentation or good information about the structure of the problem".

In this paper, we apply the Least-Squares Monte Carlo method intensively to price four different American Asian (Amerasian) options with four different polynomial basis. We do this for both put and call option. To access the performance of this algorithm, we use three sets of parameters.

\section{The Least-Squares Monte Carlo Method}

Every minute prior to the expiration date of an American option, the option holder must choose the optimal outcome between exercising the option or keeping it for another term. The choice is based on the immediate exercise payoff and the future payoff expected. If the former is greater than the latter, then the option is exercised. 
Consistent with the no-arbitrage valuation theory, the continuation value is the riskneutral expectation of the discounted future cash flow, i.e.,:

$$
F\left(\omega ; t_{k}\right)=E^{Q}\left[\sum_{j=k+1}^{K} \exp \left(-\int_{t_{k}}^{t_{j}} r(\omega, s) d s\right) C\left(\omega, t_{j} ; t_{k}, T\right) \mid \mathfrak{I}_{t_{k}}\right]
$$

Where,

$F\left(\omega ; t_{k}\right) \equiv$ continuation value at time $t_{k}$ on sample path $\omega ;$

$E^{Q}\left[. \mid \mathfrak{I}_{t_{k}}\right] \equiv$ risk-neutral expectation conditional on $\mathfrak{I}_{t_{k}}$;

$Q \equiv$ risk-neutral probability measure;

$r(\omega, s) \equiv$ riskless discount rate;

$C\left(\omega, t_{j} ; t_{k}, T\right) \equiv$ cash flows generated by the option conditional

on the option not being exercised at or prior to $t_{k}$ and on the option holder

following the optimal stopping strategy for all $t_{j}, t_{k}<t_{j} \leq T$; and

$\mathfrak{S}_{t_{k}} \equiv$ information set at time $t_{k}$.

The idea of the Least-Squares Monte Carlo Method is to approximate the continuation value by using least-squares regression at every moment in which it is possible to exercise the option. The regression is done based on the data obtained for the state variables via Monte Carlo simulation and by choosing the trajectories where the option is in the money.

Within this context, whenever it is possible to exercise the option, the continuation value can be expressed as a linear combination of orthogonal basis functions, such as Power, Legendre and Laguerre polynomials. This follows from the Finance Literature that considers the payoff functions that belong to the function space of finite variance, represented by $L^{2}(\Omega, \mathfrak{I}, Q){ }^{1}$. Since this is a Hilbert space ${ }^{2}$, any function $F$ that belongs to this space can be written as a linear combination of orthogonal basis functions (Lima 2007); (Stentoft 2004). Thus, the same function $F$ can be rewritten as:

$$
F\left(\omega ; t_{k}\right)=\sum_{j=0}^{N} \alpha_{J} \Phi_{J}, \alpha_{J} \in \mathbb{R}
$$

where the polynomial basis, represented by $\Phi_{j}$, is a function of one of the state variables. Note that the coefficients of the basis, $\alpha_{j}$, are not previously known, but they can be estimated by linear regression. The properties of the estimator and the convergence of the method are addressed algebraically by Clement et al. (2002) and Stentoft (2004).

When considering the number of basis to be used, it is important to point out a problem mentioned by Moreno and Navas (2003). They analyze the use of a large number of basis and conclude that several degrees of the chosen polynomial basis and its respective crossed products can increase the accuracy of the estimation. In some cases, however, an excessive increase in the number of basis can reduce the precision of the method, making it computationally expensive.

\section{Methods}

The Least-Squares Monte Carlo Method is applied in four different cases of Fixed-Start Time Window ${ }^{3}$ American Asian options: Case 1 -Arithmetic Average Floating Strike 
American Asian option; Case 2 -Geometric Average Floating Strike American Asian Option; Case 3 -Arithmetic Average Fixed Strike American Asian Option; and Case 4 Geometric Average Fixed Strike American Asian Option.

Following Moreno and Navas (2003), we use the Bermuda-Asian option as a discrete approximation of the Amerasian option, whereby the price of the asset-object is monthly noted. All the priced options have a term of one year, and can be exercised from the third month of their issuance. It is assumed that the price of the asset-object follows a risk-neutral Geometric Brownian Motion.

Based on matrix notation, in which every column represents a specific time step and every line represents a simulated path, their respective payoff functions are formally expressed below, and the variables are set according to the following definitions:

$t_{j} \equiv j$ represents month in which early exercise is possible, $j=1,2, \ldots, 12$.

$S\left(t_{j}\right)=S_{j} \equiv$ underlying asset price observed at $j$ th month

$\bar{S}\left(t_{j}\right)=\bar{S}_{j} \equiv$ discretely sampled average of underlying asset price

$\bar{S}_{j}=A_{j} \equiv \frac{\sum_{k=1}^{j} S_{k}}{j} \Rightarrow$ discretely sampled arithmetic average

$\bar{S}_{j}=G_{j} \equiv\left(\prod_{k=1}^{j} S_{k}\right)^{1 / j} \Rightarrow$ discretely sampled geometric average

$K \equiv$ strike price

$\eta=\left\{\begin{array}{c}1 \text { for call option, } \\ -1 \text { for put option. }\end{array}\right.$

$y \equiv$ dividend yield

$r \equiv$ riskless rate

$\sigma \equiv$ volatility

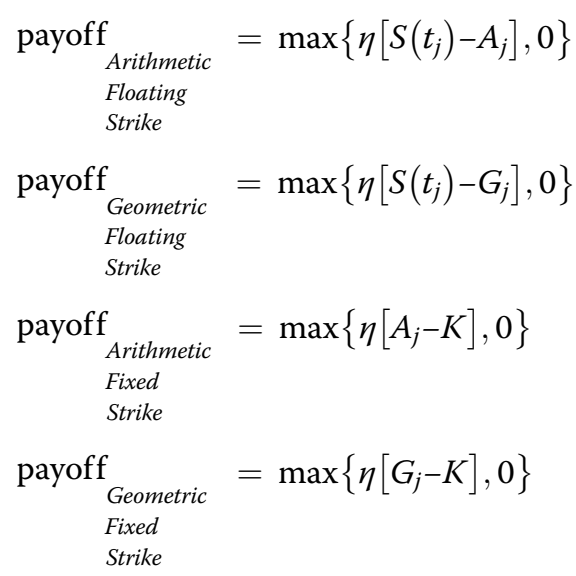

According to Longstaff and Schwartz (2001), the structure used in regressions, expressed below by equation (1), comprises a constant, the two first degrees of the chosen polynomial basis and their crossed product up to the third degree, totaling eight basis. The polynomials used are Power, Legendre, Laguerre and Hermite A.

The matrix of the underlying asset prices, $\mathbf{S}$, is defined recursively. The $i^{\text {th }}$ row vector corresponds to the $i^{\text {th }}$ simulated path and its elements are defined by: 


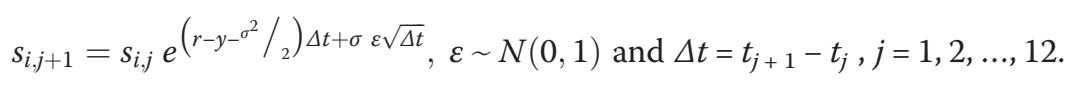

Note that each column vector of the matrix corresponds to a specific time step.

Once the matrix of the underlying asset prices is set, the matrix of averages, $\overline{\mathbf{S}}$, is determined by using relations introduced latter, in which the form depends solely on what kind of average is considered, i.e., whether it is an arithmetic average or it is a geometric one.

Let,

$$
\begin{aligned}
& \Phi_{1}(\mathbf{S}) \equiv \mathbf{B}_{m, j} ; \Phi_{2}(\mathbf{S}) \equiv \mathbf{C}_{m, j} ; \Phi_{1}(\overline{\mathbf{S}}) \equiv \mathbf{D}_{m, j} ; \Phi_{2}(\overline{\mathbf{S}}) \equiv \mathbf{E}_{m, j} ; \\
& \Phi_{1}(\mathbf{S}) \odot \Phi_{1}(\overline{\mathbf{S}}) \equiv \mathbf{Z}_{m, j} ; \Phi_{2}(\mathbf{S}) \odot \Phi_{1}(\overline{\mathbf{S}}) \equiv \mathbf{G}_{m, j} ; \Phi_{1}(\mathbf{S}) \odot \Phi_{2}(\overline{\mathbf{S}}) \equiv \mathbf{H}_{m, j}
\end{aligned}
$$

where, $\Phi_{n}($.$) is the polynomial basis of n^{\text {th }}$ degree and it is a function of one of the state variables. The dimension of matrices is set by $j$ time steps and $m$ simulated paths. So, at $j^{\text {th }}$ time step, the regression is performed by using the column vector related to the same time step. Therefore,

$$
F\left(\mathbf{S}_{j}, \overline{\mathbf{S}}_{j}\right)=\alpha_{1}+\alpha_{2} \cdot \mathbf{b}_{j}+\alpha_{3} \cdot \mathbf{c}_{j}+\alpha_{4} \cdot \mathbf{d}_{j}+\alpha_{5} \cdot \mathbf{e}_{j}+\alpha_{6} \cdot \mathbf{z}_{j}+\alpha_{7} \cdot \mathbf{g}_{j}+\alpha_{8} \cdot \mathbf{h}_{j}
$$

In this work, the Monte Carlo simulation was performed with pseudorandom number sequences and the variance reduction technique used is antithetic variables ${ }^{4}$. We used three sets of parameters varying them solely on strike prices used in experiments as follows:

$$
\begin{aligned}
& S\left(t_{1}\right)=100, K=95, \sigma=20 \%, \mathrm{r}=5 \%, \mathrm{y}=0 ; \\
& S\left(t_{1}\right)=K=100, \sigma=20 \%, \mathrm{r}=5 \%, \mathrm{y}=0
\end{aligned}
$$

and

$$
S\left(t_{1}\right)=100, K=105, \sigma=20 \%, \mathrm{r}=5 \%, \mathrm{y}=0 .
$$

\section{Polynomial basis used}

As mentioned above, the polynomials used in this study are Power, Legendre, Laguerre and Hermite A. All of them can be alternatively expressed by Rodrigues' formula, explicit form or by the recurrence law (Abramowitz and Stegun 1972).

Following Abramowitz and Stegun (1972), Rodrigues' formula is expressed by:

$$
f_{n}(x)=\frac{1}{a_{n} \cdot \rho(x)} \cdot \frac{\partial^{n}}{\partial x^{n}}\left[\rho(x) \cdot(g(x))^{n}\right]
$$

Where $n$ is the polynomial degree $(n \geq 0)$. The coefficients and functions of Rodrigues' formula for each of the polynomials are detailed in Table 1.

Alternatively, it is possible to use the explicit form, whose terms are specified in Table 2, to represent the polynomials:

Table 1 Coefficients and functions of the basis functions using Rodrigues' formula

\begin{tabular}{lllll}
\hline & $f_{n}(x)$ & $a_{n}$ & $\rho(x)$ & $g(x)$ \\
\hline Power & $W_{n}(x)$ & $(2 \cdot n) ! / n !$ & $x^{2 \cdot n}$ & 1 \\
Legendre & $P_{n}(x)$ & $(-1)^{n} \cdot 2^{n} \cdot n !$ & 1 & $1-x^{2}$ \\
Laguerre & $L_{n}(x)$ & $n !$ & $e^{-x}$ & $x$ \\
Hermite A & $H_{n}(x)$ & $(-1)^{n}$ & $e^{-x^{2}}$ & 1 \\
\hline
\end{tabular}


Table 2 Explicit expressions of the basis functions

\begin{tabular}{llllll}
\hline & $f_{n}(x)$ & $N$ & $d_{n}$ & $c_{m}$ & $g_{m}(x)$ \\
\hline Power & $W_{n}(x)$ & 0 & 1 & 1 & $x^{n}$ \\
Legendre & $P_{n}(x)$ & {$[n / 2]$} & $2^{-n}$ & $(-1)^{m} \cdot\left(\begin{array}{l}n \\
m\end{array}\right) \cdot\left(\begin{array}{l}2 . n-2 \cdot m \\
n\end{array}\right)$ & $x^{n-2 \cdot m}$ \\
Laguerre & $L_{n}(x)$ & $n$ & 1 & $\frac{(-1)^{m}}{m !} \cdot\left(\begin{array}{l}n \\
n-m\end{array}\right)$ & $x^{m}$ \\
Hermite A & $H_{n}(x)$ & {$\left[\begin{array}{l}n \\
\text { Her }\end{array}\right]$} & $n !$ & $(-1)^{m} \cdot \frac{1}{m !(n-2 . m) !}$ & $(2 \cdot x)^{n-2 \cdot m}$ \\
\hline
\end{tabular}

$$
f_{n}=d_{n} \sum_{m=0}^{N} c_{m} \cdot g_{m}(x)
$$

The recurrence law can also be used to express the polynomials and it is described as:

$$
a_{n+1} \cdot f_{n+1}(x)=\left(a_{n}+b_{n} \cdot x\right) \cdot f_{n}(x)-a_{n-1} \cdot f_{n-1}(x)
$$

Table 3 specifies every term of the recurrence law.

The format used to write a polynomial is chosen in such a way that the pricing procedure becomes operationally more practical. This method proved to be effective throughout the whole process, excluding the possibility that the rank of the coefficient matrix of the least square regression is insufficient. Thus, the explicit form of the Power and Laguerre polynomials is applied, the recurrence law is used to express the Hermite A polynomial, and Rodrigues' formula is used to write the Legendre polynomial.

\section{Results and discussion}

Tables 4, 5, 6 and 7 show the average prices and their respective standard deviations of call and put options for the four types of Amerasian options studied. Tables 4, 5, 6 and 7 show that when different basis are used in all sets of simulated trajectories, and taking into account all sets of parameters used, there is a slight difference among the estimates of the option price. These results follow what Moreno and Navas (2003) stated i.e., the choice of the polynomial basis affects the estimates of the option prices. This difference becomes insignificant when a greater number of simulated trajectories is used.

Such pattern is the same for both put options and for call options of all the American Asian options considered. This result suggests two basic conclusions. First, it ratifies one of the main features of the method: if all of the polynomial basis are orthogonal, then theoretically all of them could be indistinctly used in derivative pricing. Second, it shows that the gradual reduction of differences among the price estimates for each of the basis due to the incremental increase in the number of simulated paths is a direct result of the law of large numbers.

Table 3 Recurrence law for the basis functions reported

\begin{tabular}{llllllll}
\hline & $f_{n}(x)$ & $a_{n+1}$ & $a_{n}$ & $b_{n}$ & $a_{n-1}$ & $f_{0}(x)$ & $f_{1}(x)$ \\
\hline Power & $W_{n}(x)$ & 1 & 0 & 1 & 0 & 1 & $x$ \\
Legendre & $P_{n}(x)$ & $n+1$ & 0 & $2 \cdot n+1$ & $n$ & 1 & $x$ \\
Laguerre & $L_{n}(x)$ & $n+1$ & $2 \cdot n+1$ & -1 & $n$ & 1 & $1-x$ \\
Hermite A & $H_{n}(x)$ & 1 & 0 & 2 & $2 . n$ & 1 & $2 . x$ \\
\hline
\end{tabular}


Table 4 Case 1: Estimated prices for different set of simulated paths

\begin{tabular}{|c|c|c|c|c|c|c|}
\hline \multicolumn{7}{|c|}{ Case 1 - Arithmetic floating strike } \\
\hline PUT - Strike 105 & 100 & 500 & 1000 & 5000 & 10000 & 50000 \\
\hline Power & $5,5292(0,38652)$ & $5,1195(0,12007)$ & $5,0274(0,0677)$ & $4,9441(0,0346)$ & $4,9519(0,03081)$ & $4,9541(0,01721)$ \\
\hline Legendre & $5,5277(0,37698)$ & $5,0311(0,11412)$ & $5,0073(0,10189)$ & $4,9647(0,05079)$ & $4,9573(0,03045)$ & $4,9511(0,01641)$ \\
\hline Laguerre & $5,4596(0,26394)$ & $5,1052(0,24922)$ & $5,0131(0,07685)$ & $4,9642(0,0636)$ & $4,9631(0,02601)$ & $4,9566(0,01498)$ \\
\hline Hermite A & $5,2566(0,30584)$ & $5,0787(0,14616)$ & $5,0821(0,10896)$ & $4,9606(0,04224)$ & $4,9645(0,04333)$ & $4,9566(0,01496)$ \\
\hline PUT - Strike 100 & 100 & 500 & 1000 & 5000 & 10000 & 50000 \\
\hline Power & $4,8737(0,25913)$ & $4,392(0,14042)$ & $4,4421(0,08279)$ & $4,3984(0,03246)$ & $4,3822(0,03117)$ & $4,3898(0,01394)$ \\
\hline Legendre & $4,8186(0,24198)$ & $4,5005(0,1226)$ & $4,5409(0,0741)$ & $4,4328(0,04683)$ & 4,395 $(0,025)$ & $4,3905(0,01458)$ \\
\hline Laguerre & $4,8157(0,26914)$ & $4,4182(0,14124)$ & 4,4109 $(0,07254)$ & 4,394 $(0,03582)$ & 4,3984 $(0,01837)$ & 4,3906 $(0,01461)$ \\
\hline Hermite A & $4,8801(0,26384)$ & $4,5168(0,12988)$ & $4,4292(0,0761)$ & $4,3965(0,04536)$ & $4,3969(0,02133)$ & $4,3905(0,01195)$ \\
\hline PUT - Strike 95 & 100 & 500 & 1000 & 5000 & 10000 & 50000 \\
\hline Power & $4,887(0,44331)$ & $4,5837(0,10469)$ & $4,5932(0,10125)$ & $4,4892(0,05058)$ & $4,4924(0,02487)$ & $4,4837(0,01323)$ \\
\hline Legendre & $4,905(0,30647)$ & $4,6709(0,10534)$ & $4,5098(0,08731)$ & $4,5039(0,04946)$ & $4,4732(0,02929)$ & 4,4846 $(0,01352)$ \\
\hline Laguerre & $5,0175(0,30984)$ & $4,565(0,11335)$ & $4,5149(0,09689)$ & 4,49 $(0,02836)$ & $4,4904(0,02779)$ & $4,4846(0,01338)$ \\
\hline Hermite A & $5,011(0,27431)$ & $4,6248(0,14327)$ & $4,5676(0,12363)$ & $4,4965(0,04904)$ & $4,4732(0,02929)$ & 4,4846 $(0,01352)$ \\
\hline CALL - Strike 105 & 100 & 500 & 1000 & 5000 & 10000 & 50000 \\
\hline Power & $3,1783(0,24351)$ & $3,2916(0,11733)$ & $3,3286(0,07488)$ & $3,2771(0,03209)$ & $3,2903(0,01621)$ & $3,2758(0,01799)$ \\
\hline Legendre & $3,1421(0,26638)$ & $3,2268(0,11876)$ & $3,2538(0,04496)$ & $3,265(0,03664)$ & $3,2888(0,01995)$ & $3,2818(0,01131)$ \\
\hline Laguerre & $3,2969(0,2)$ & $3,2741(0,15825)$ & $3,2853(0,09247)$ & $3,2713(0,0395)$ & $3,2859(0,02331)$ & $3,2764(0,01669)$ \\
\hline Hermite A & $3,3147(0,30627)$ & $3,2757(0,10231)$ & $3,2679(0,06761)$ & $3,2849(0,02703)$ & $3,2796(0,03082)$ & $3,2764(0,01668)$ \\
\hline CALL - Strike 100 & 100 & 500 & 1000 & 5000 & 10000 & 50000 \\
\hline Power & $3,0867(0,38686)$ & $2,984(0,1241)$ & $3,0561(0,07181)$ & $3,0186(0,03374)$ & $2,9977(0,03123)$ & $3,0122(0,01231)$ \\
\hline Legendre & $3,0231(0,35521)$ & $3,0533(0,12091)$ & $3,0219(0,08862)$ & $3,0323(0,03966)$ & $3,0275(0,02892)$ & $3,0125(0,01349)$ \\
\hline Laguerre & $3,1118(0,38252)$ & $2,9581(0,13003)$ & $3,0992(0,07227)$ & $3,0287(0,03935)$ & $3,0127(0,02573)$ & $3,0126(0,01371)$ \\
\hline Hermite A & $2,9222(0,3244)$ & $3,0657(0,12064)$ & $3,0454(0,08328)$ & $3,0199(0,05572)$ & $3,0092(0,03606)$ & $3,0156(0,015)$ \\
\hline CALL - Strike 95 & 100 & 500 & 1000 & 5000 & 10000 & 50000 \\
\hline Power & $3,085(0,23395)$ & $2,9682(0,16596)$ & $3,0049(0,05549)$ & $2,9916(0,03029)$ & $2,9789(0,02461)$ & $2,9641(0,01496)$ \\
\hline Legendre & $2,8932(0,2631)$ & $3,0064(0,12606)$ & $2,987(0,10273)$ & $2,9663(0,05163)$ & $2,9482(0,01573)$ & $2,9644(0,01507)$ \\
\hline Laguerre & $2,8498(0,26982)$ & $2,9404(0,11433)$ & $2,9354(0,07099)$ & $2,9595(0,03359)$ & $2,9694(0,02955)$ & $2,9644(0,01508)$ \\
\hline Hermite A & $2,8649(0,25414)$ & $2,9375(0,15739)$ & $2,9957(0,08331)$ & $2,9702(0,04905)$ & $2,9482(0,01573)$ & $2,9644(0,01507)$ \\
\hline
\end{tabular}

Standard deviations in parentheses. Simulated path (NumSim) throughout all sets of parameters i.e., $K=95, K=100, K=105$ 
Table 5 Case 2: Estimated prices for different set of simulated paths

\begin{tabular}{|c|c|c|c|c|c|c|}
\hline \multicolumn{7}{|c|}{ Case 2 - Geometric floating strike } \\
\hline PUT - Strike 105 & 100 & 500 & 1000 & 5000 & 10000 & 50000 \\
\hline Power & $5,4008(0,40284)$ & $5,0057(0,11024)$ & $4,9288(0,07604)$ & $4,8491(0,04653)$ & $4,852(0,03007)$ & $4,8519(0,01425)$ \\
\hline Legendre & $5,3411(0,33629)$ & $4,9315(0,12912)$ & $4,8444(0,09204)$ & $4,8634(0,05167)$ & $4,8612(0,05081)$ & $4,8526(0,0147)$ \\
\hline Laguerre & $5,5355(0,20011)$ & $4,8723(0,15434)$ & $4,8561(0,0866)$ & $4,8863(0,04501)$ & $4,8703(0,0353)$ & $4,8529(0,01454)$ \\
\hline Hermite A & $5,3667(0,41746)$ & $4,9731(0,13953)$ & $4,9061(0,08725)$ & $4,8608(0,04106)$ & $4,858(0,03583)$ & $4,8524(0,01237)$ \\
\hline PUT - Strike 100 & 100 & 500 & 1000 & 5000 & 10000 & 50000 \\
\hline Power & $4,8737(0,2144)$ & 4,392 (0,13649) & 4,4421 $(0,0691)$ & $4,3984(0,03888)$ & $4,3822(0,02424)$ & $4,3898(0,01473)$ \\
\hline Legendre & $4,8186(0,27618)$ & $4,5005(0,08995)$ & $4,5409(0,10719)$ & $4,4328(0,03967)$ & $4,395(0,02285)$ & 4,3905 $(0,01474)$ \\
\hline Laguerre & $4,8157(0,18517)$ & $4,4182(0,13916)$ & $4,4109(0,07725)$ & 4,394 (0,04539) & $4,3984(0,02433)$ & $4,3906(0,01433)$ \\
\hline Hermite A & $4,8801(0,20338)$ & $4,5168(0,16687)$ & $4,4292(0,07855)$ & $4,3965(0,03733)$ & $4,3969(0,02125)$ & $4,3905(0,01342)$ \\
\hline PUT - Strike 95 & 100 & 500 & 1000 & 5000 & 10000 & 50000 \\
\hline Power & 4,8737 $(0,2946)$ & 4,392 (0,15808) & $4,4421(0,07126)$ & $4,3984(0,04749)$ & $4,3822(0,02766)$ & 4,3898 $(0,0129)$ \\
\hline Legendre & $4,8186(0,21728)$ & $4,5005(0,13473)$ & $4,5409(0,08409)$ & $4,4328(0,03236)$ & 4,395 (0,03323) & $4,3905(0,01331)$ \\
\hline Laguerre & $4,8157(0,20457)$ & $4,4182(0,11763)$ & $4,4109(0,06621)$ & $4,394(0,03963)$ & $4,3984(0,02963)$ & $4,3906(0,01332)$ \\
\hline Hermite A & $4,8801(0,54061)$ & $4,5168(0,19166)$ & $4,4292(0,11576)$ & $4,3965(0,05203)$ & $4,3969(0,04185)$ & $4,3905(0,01331)$ \\
\hline CALL - Strike 105 & 100 & 500 & 1000 & 5000 & 10000 & 50000 \\
\hline Power & $3,2097(0,22214)$ & $3,3416(0,12657)$ & $3,3249(0,09254)$ & $3,336(0,03828)$ & $3,324(0,03941)$ & $3,3293(0,01685)$ \\
\hline Legendre & $3,214(0,28978)$ & $3,3615(0,14556)$ & $3,3733(0,09039)$ & $3,321(0,04469)$ & $3,3486(0,0313)$ & $3,3296(0,01689)$ \\
\hline Laguerre & $3,252(0,28853)$ & $3,3193(0,13172)$ & $3,354(0,10208)$ & $3,3189(0,02632)$ & 3,3495 (0,02309) & $3,3297(0,01692)$ \\
\hline Hermite A & $3,3304(0,21868)$ & $3,4262(0,14675)$ & $3,2753(0,07181)$ & $3,3237(0,05071)$ & $3,3376(0,02572)$ & $3,3297(0,01661)$ \\
\hline CALL - Strike 100 & 100 & 500 & 1000 & 5000 & 10000 & 50000 \\
\hline Power & $3,0867(0,35108)$ & $2,984(0,13725)$ & $3,0561(0,09443)$ & $3,0186(0,03458)$ & $2,9977(0,02952)$ & $3,0122(0,01336)$ \\
\hline Legendre & $3,0231(0,27413)$ & $3,0533(0,09543)$ & $3,0219(0,08744)$ & $3,0323(0,04844)$ & $3,0275(0,03365)$ & $3,0125(0,01356)$ \\
\hline Laguerre & $3,1118(0,31237)$ & $2,9581(0,15785)$ & $3,0992(0,08248)$ & $3,0287(0,04877)$ & $3,0127(0,03003)$ & $3,0126(0,01666)$ \\
\hline Hermite A & $2,9222(0,35909)$ & $3,0657(0,1357)$ & $3,0454(0,05049)$ & $3,0199(0,03075)$ & $3,0092(0,02814)$ & $3,0156(0,01356)$ \\
\hline CALL - Strike 95 & 100 & 500 & 1000 & 5000 & 10000 & 50000 \\
\hline Power & $3,0867(0,2807)$ & $2,984(0,07714)$ & $3,0561(0,069)$ & $3,0186(0,05047)$ & $2,9977(0,01636)$ & $3,0122(0,01525)$ \\
\hline Legendre & $3,0231(0,24697)$ & $3,0533(0,14612)$ & $3,0219(0,09397)$ & $3,0323(0,02256)$ & $3,0275(0,02232)$ & $3,0125(0,01527)$ \\
\hline Laguerre & $3,1118(0,22394)$ & $2,9581(0,09095)$ & $3,0992(0,09578)$ & $3,0287(0,02069)$ & $3,0127(0,02792)$ & $3,0126(0,01531)$ \\
\hline Hermite A & $2,9222(0,2811)$ & $3,0657(0,08468)$ & $3,0454(0,09731)$ & $3,0199(0,0453)$ & $3,0092(0,01941)$ & $3,0156(0,01422)$ \\
\hline
\end{tabular}

Standard deviations in parentheses. Simulated path (NumSim) throughout all sets of parameters i.e., $K=95, K=100, K=105$ 
Table 6 Case 3: Estimated prices for different set of simulated paths

\begin{tabular}{|c|c|c|c|c|c|c|}
\hline \multicolumn{7}{|c|}{ Case 3 - Arithmetic fixed strike } \\
\hline PUT - Strike 105 & 100 & 500 & 1000 & 5000 & 10000 & 50000 \\
\hline Power & $6,7237(0,33767)$ & $6,7068(0,10996)$ & $6,6948(0,07619)$ & $6,6841(0,05154)$ & $6,6669(0,05266)$ & $6,6692(0,02473)$ \\
\hline Legendre & $6,6299(0,25683)$ & $6,7175(0,11842)$ & $6,636(0,0711)$ & $6,6629(0,04606)$ & $6,6631(0,0545)$ & $6,6622(0,02162)$ \\
\hline Laguerre & $6,7273(0,34734)$ & $6,6902(0,10582)$ & $6,7413(0,09639)$ & $6,6739(0,0597)$ & $6,666(0,05421)$ & $6,6622(0,02166)$ \\
\hline Hermite A & $6,7695(0,37345)$ & $6,7103(0,09924)$ & $6,7262(0,17196)$ & $6,6625(0,04541)$ & $6,6481(0,02537)$ & $6,6622(0,02167)$ \\
\hline PUT - Strike 100 & 100 & 500 & 1000 & 5000 & 10000 & 50000 \\
\hline Power & $4,8737(0,20587)$ & 4,392 $(0,08289)$ & $4,4421(0,05145)$ & $4,3984(0,02722)$ & $4,3822(0,01973)$ & $4,3898(0,0101)$ \\
\hline Legendre & $4,8186(0,22622)$ & $4,5005(0,08516)$ & $4,5409(0,06124)$ & $4,4328(0,02677)$ & 4,395 (0,02303) & 4,3905 (0,01009) \\
\hline Laguerre & $4,8157(0,18574)$ & $4,4182(0,07473)$ & $4,4109(0,04725)$ & $4,394(0,03594)$ & 4,3984 (0,01532) & 4,3906 $(0,01004)$ \\
\hline Hermite A & $4,8801(0,19166)$ & $4,5168(0,08783)$ & $4,4292(0,0467)$ & $4,3965(0,02859)$ & $4,3969(0,01286)$ & 4,3905 (0,01001) \\
\hline PUT - Strike 95 & 100 & 500 & 1000 & 5000 & 10000 & 50000 \\
\hline Power & $1,5292(0,23719)$ & $1,6134(0,15668)$ & $1,6079(0,10196)$ & $1,5839(0,02465)$ & $1,5744(0,01738)$ & $1,5612(0,01605)$ \\
\hline Legendre & $1,6529(0,24375)$ & $1,5715(0,09924)$ & $1,5483(0,06678)$ & $1,5543(0,03563)$ & $1,5712(0,02131)$ & $1,5609(0,0159)$ \\
\hline Laguerre & $1,66(0,30421)$ & $1,5603(0,09967)$ & $1,6074(0,11454)$ & $1,5781(0,02878)$ & $1,5747(0,01671)$ & $1,5609(0,0159)$ \\
\hline Hermite A & $1,6873(0,35452)$ & $1,5603(0,09967)$ & $1,6074(0,11454)$ & $1,5781(0,02882)$ & $1,5747(0,01667)$ & $1,5609(0,0159)$ \\
\hline CALL - Strike 105 & 100 & 500 & 1000 & 5000 & 10000 & 50000 \\
\hline Power & $0,6458(0,07247)$ & $0,6472(0,0309)$ & $0,655(0,02674)$ & $0,6554(0,02315)$ & $0,6488(0,01533)$ & $0,6547(0,00766)$ \\
\hline Legendre & $0,6166(0,14273)$ & $0,6607(0,03518)$ & $0,662(0,03774)$ & $0,6557(0,01901)$ & $0,6524(0,01889)$ & $0,6539(0,00705)$ \\
\hline Laguerre & $0,5946(0,08017)$ & $0,6477(0,04393)$ & $0,6554(0,0448)$ & $0,6585(0,02542)$ & $0,649(0,01538)$ & $0,6539(0,00705)$ \\
\hline Hermite A & $0,6286(0,10374)$ & $0,6688(0,04487)$ & $0,6663(0,02847)$ & $0,6532(0,01512)$ & $0,6508(0,01129)$ & $0,6539(0,00705)$ \\
\hline CALL - Strike 100 & 100 & 500 & 1000 & 5000 & 10000 & 50000 \\
\hline Power & $3,0867(0,37492)$ & $2,984(0,10728)$ & $3,0561(0,0726)$ & $3,0186(0,06132)$ & $2,9977(0,04801)$ & $3,0122(0,01715)$ \\
\hline Legendre & $3,0231(0,35095)$ & $3,0533(0,13885)$ & $3,0219(0,08161)$ & $3,0323(0,05333)$ & $3,0275(0,04622)$ & $3,0125(0,01748)$ \\
\hline Laguerre & $3,1118(0,31191)$ & $2,9581(0,1255)$ & $3,0992(0,09784)$ & $3,0287(0,066)$ & $3,0127(0,03268)$ & $3,0126(0,01796)$ \\
\hline Hermite A & $2,9222(0,35167)$ & $3,0657(0,16015)$ & $3,0454(0,0979)$ & $3,0199(0,05945)$ & $3,0092(0,03579)$ & $3,0156(0,01795)$ \\
\hline CALL - Strike 95 & 100 & 500 & 1000 & 5000 & 10000 & 50000 \\
\hline Power & $5,5638(0,06386)$ & $5,5919(0,04907)$ & $5,5676(0,03613)$ & $5,5884(0,01042)$ & $5,5865(0,00963)$ & $5,5795(0,00573)$ \\
\hline Legendre & $5,5388(0,06966)$ & $5,5773(0,02321)$ & $5,5956(0,03641)$ & $5,5818(0,0193)$ & $5,5825(0,01016)$ & $5,5795(0,00576)$ \\
\hline Laguerre & $5,617(0,11439)$ & $5,5729(0,04111)$ & $5,5777(0,03414)$ & $5,5875(0,01177)$ & $5,5865(0,00956)$ & $5,5795(0,00576)$ \\
\hline Hermite A & $5,6234(0,10946)$ & $5,5729(0,04111)$ & $5,5777(0,03414)$ & $5,5875(0,01177)$ & $5,5865(0,00956)$ & $5,5795(0,00576)$ \\
\hline
\end{tabular}

Standard deviations in parentheses. Simulated path (NumSim) throughout all sets of parameters i.e., $K=95, K=100, K=105$ 
Table 7 Case 4: Estimated prices for different set of simulated paths

\begin{tabular}{|c|c|c|c|c|c|c|}
\hline \multicolumn{7}{|c|}{ Case 4 - Geometric fixed strike } \\
\hline PUT - Strike 105 & 100 & 500 & 1000 & 5000 & 10000 & 50000 \\
\hline Power & $6,8045(0,23302)$ & $6,8008(0,13203)$ & $6,7869(0,07603)$ & $6,7925(0,05448)$ & $6,7882(0,03496)$ & $6,801(0,02277)$ \\
\hline Legendre & $6,7919(0,32414)$ & $6,8177(0,15514)$ & $6,8438(0,05622)$ & $6,8042(0,06049)$ & $6,7796(0,04728)$ & $6,7942(0,02165)$ \\
\hline Laguerre & $6,9194(0,20012)$ & $6,838(0,14021)$ & $6,8201(0,07885)$ & $6,8014(0,05276)$ & $6,8106(0,03512)$ & $6,7942(0,02156)$ \\
\hline Hermite A & $6,8552(0,26187)$ & $6,8255(0,09763)$ & $6,8773(0,11303)$ & $6,809(0,04093)$ & $6,7957(0,03321)$ & $6,7967(0,02055)$ \\
\hline PUT - Strike 100 & 100 & 500 & 1000 & 5000 & 10000 & 50000 \\
\hline Power & $4,8737(0,19727)$ & 4,392 $(0,10796)$ & $4,4421(0,04911)$ & $4,3984(0,03353)$ & 4,3822 (0,01958) & 4,3898 $(0,00954)$ \\
\hline Legendre & $4,8186(0,15306)$ & $4,5005(0,05025)$ & $4,5409(0,04643)$ & $4,4328(0,02551)$ & $4,395(0,01771)$ & $4,3905(0,0067)$ \\
\hline Laguerre & $4,8157(0,21805)$ & $4,4182(0,07679)$ & $4,4109(0,04758)$ & $4,394(0,0257)$ & $4,3984(0,02187)$ & $4,3906(0,00958)$ \\
\hline Hermite A & $4,8801(0,22004)$ & $4,5168(0,07318)$ & $4,4292(0,04935)$ & $4,3965(0,02862)$ & $4,3969(0,0216)$ & 4,3905 (0,00917) \\
\hline PUT - Strike 95 & 100 & 500 & 1000 & 5000 & 10000 & 50000 \\
\hline Power & $1,7424(0,32208)$ & $1,6518(0,10318)$ & $1,6974(0,12042)$ & $1,6701(0,03038)$ & $1,6648(0,01796)$ & $1,6514(0,01658)$ \\
\hline Legendre & $1,6857(0,42331)$ & $1,7019(0,17981)$ & $1,6196(0,08402)$ & $1,6541(0,03042)$ & $1,6618(0,02214)$ & $1,6512(0,01646)$ \\
\hline Laguerre & $1,7466(0,32841)$ & $1,6519(0,10429)$ & $1,697(0,11716)$ & $1,6682(0,02973)$ & $1,6652(0,01772)$ & $1,6512(0,01645)$ \\
\hline Hermite A & $1,7466(0,32841)$ & $1,6519(0,10429)$ & $1,697(0,11716)$ & $1,6682(0,02974)$ & $1,6651(0,01771)$ & $1,6512(0,01646)$ \\
\hline CALL - Strike 105 & 100 & 500 & 1000 & 5000 & 10000 & 50000 \\
\hline Power & $0,5694(0,11528)$ & $0,6052(0,02571)$ & $0,6195(0,0231)$ & $0,6091(0,02097)$ & $0,609(0,00636)$ & $0,6107(0,00642)$ \\
\hline Legendre & $0,5937(0,12399)$ & $0,6198(0,05949)$ & $0,6181(0,04114)$ & $0,6153(0,01535)$ & $0,6111(0,01035)$ & $0,6096(0,00624)$ \\
\hline Laguerre & $0,5683(0,08619)$ & $0,6195(0,03649)$ & $0,5991(0,02705)$ & $0,6056(0,01875)$ & $0,6075(0,01303)$ & $0,6096(0,00624)$ \\
\hline Hermite A & $0,639(0,08424)$ & $0,6061(0,05311)$ & $0,6229(0,03654)$ & $0,6164(0,01723)$ & $0,6094(0,01446)$ & $0,6097(0,00549)$ \\
\hline CALL - Strike 100 & 100 & 500 & 1000 & 5000 & 10000 & 50000 \\
\hline Power & $3,0867(0,28263)$ & $2,984(0,18382)$ & $3,0561(0,11169)$ & $3,0186(0,04615)$ & $2,9977(0,03817)$ & $3,0122(0,01919)$ \\
\hline Legendre & $3,0231(0,26407)$ & $3,0533(0,10516)$ & $3,0219(0,10515)$ & $3,0323(0,06786)$ & $3,0275(0,03656)$ & $3,0125(0,01501)$ \\
\hline Laguerre & $3,1118(0,39224)$ & $2,9581(0,14587)$ & $3,0992(0,09989)$ & $3,0287(0,05675)$ & $3,0127(0,03434)$ & $3,0126(0,02285)$ \\
\hline Hermite A & $2,9222(0,32296)$ & $3,0657(0,12974)$ & $3,0454(0,08487)$ & $3,0199(0,05707)$ & $3,0092(0,04068)$ & $3,0156(0,01976)$ \\
\hline CALL - Strike 95 & 100 & 500 & 1000 & 5000 & 10000 & 50000 \\
\hline Power & $5,5603(0,10979)$ & $5,5171(0,04009)$ & $5,5203(0,03478)$ & $5,5298(0,01079)$ & $5,5283(0,00947)$ & $5,5215(0,00545)$ \\
\hline Legendre & $5,4441(0,08543)$ & $5,5499(0,06434)$ & $5,5135(0,02599)$ & $5,5259(0,01888)$ & $5,5246(0,01067)$ & $5,5214(0,00543)$ \\
\hline Laguerre & $5,5604(0,10972)$ & $5,5173(0,04024)$ & $5,5202(0,03487)$ & $5,5299(0,01076)$ & $5,5282(0,00947)$ & $5,5214(0,00543)$ \\
\hline Hermite A & $5,5604(0,10972)$ & $5,5173(0,04024)$ & $5,5202(0,03487)$ & $5,5299(0,01076)$ & $5,5282(0,00947)$ & $5,5214(0,00543)$ \\
\hline
\end{tabular}

Standard deviations in parentheses. Simulated path (NumSim) throughout all sets of parameters i.e., $K=95, K=100, K=105$ 


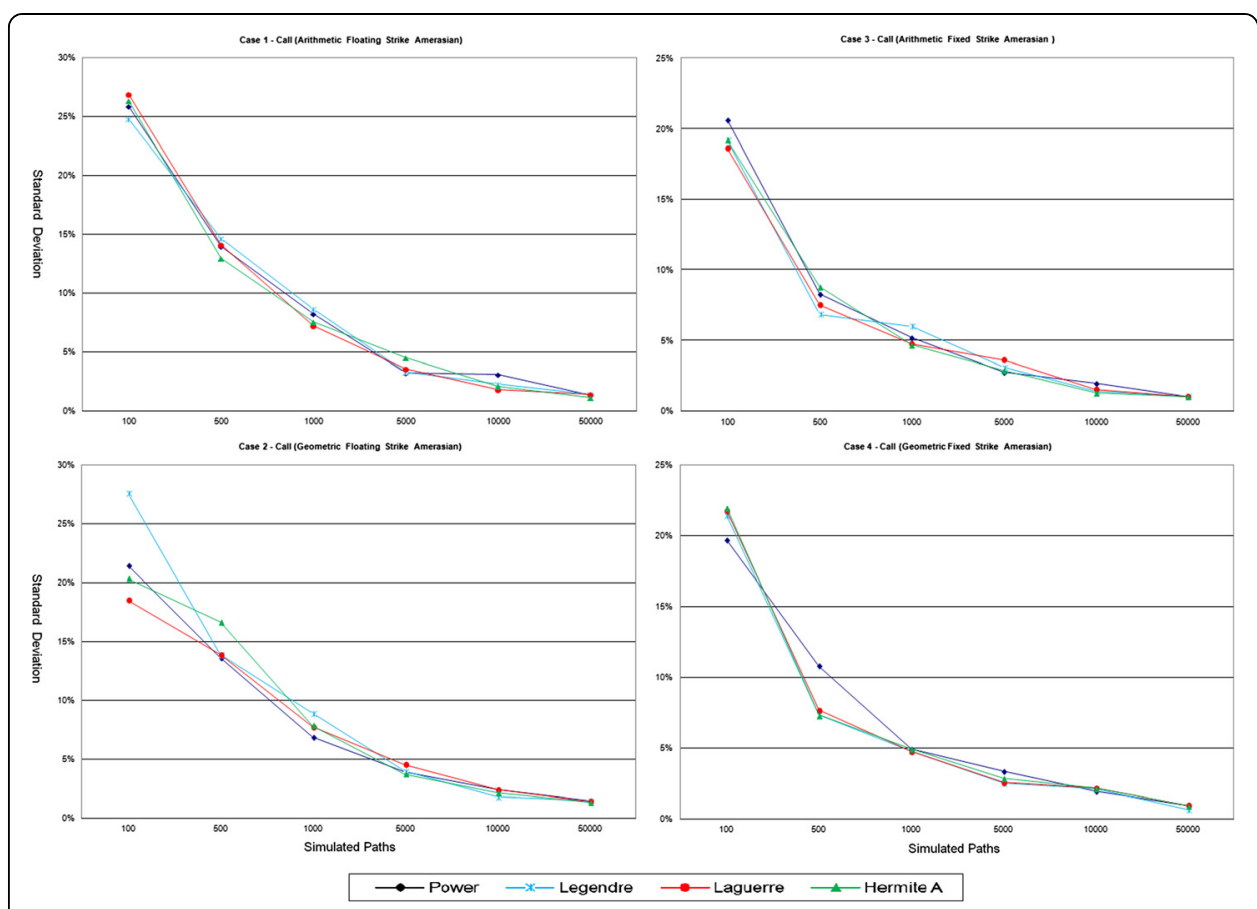

Fig. 1 Standard deviation computed over different set of simulated paths to Amerasian Call Options' cases to the following set of parameters: $S\left(t_{1}\right)=K=100 ; \sigma=20 \% ; r=5 \% ; y=0$

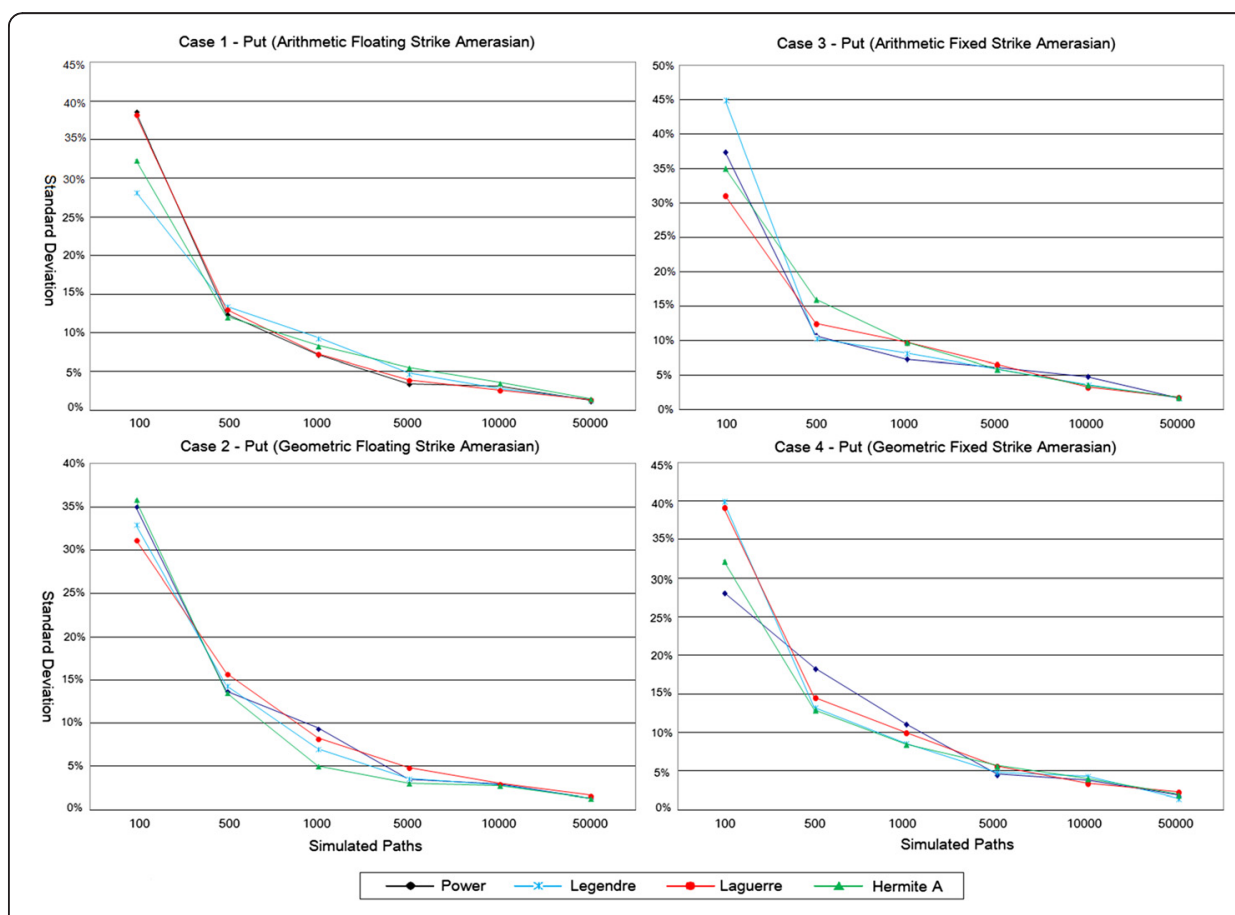

Fig. 2 Standard deviation computed over different set of simulated paths to Amerasian Put Options' cases to the following set of parameters: $S\left(t_{1}\right)=K=100 ; \sigma=20 \% ; r=5 \% ; y=0$ 
Table 8 The polynomial basis whose standard deviation is the lowest at 50.000 simulated paths

\begin{tabular}{lllll}
\hline PUT & & & & \\
Strike & Case 1 & Case 2 & Case 3 & Case 4 \\
95 & Power & Power & Legendre & Laguerre \\
100 & Hermite A & Hermite A & Hermite A & Legendre \\
105 & Hermite A & Hermite A & Legendre & Hermite A \\
CALL & & & & \\
Strike & Case 1 & Case 2 & Case 3 & Case 4 \\
95 & Power & Hermite A & Power & Legendre \\
100 & Power & Power & Power & Legendre \\
105 & Legendre & Hermite A & Legendre & Hermite A \\
\hline
\end{tabular}

The increase in the number of simulated trajectories markedly decreases the standard deviation of the price estimates for all of the basis used. Therefore, the estimates converge to their real price. This is true for both call and put American Asian options. To exemplify it graphically, Figs. 1 and 2 show the convergence of the estimated prices for the set of parameters in which $\mathrm{K}=100$ to all simulated trajectories in each studied cases considering the four polynomial basis.

The results in Table 8 suggest a certain degree of homogeneity regarding the most accurate basis considering not only the cases studied in a specific strike, but also when analyzing one case varying solely its strike price, whose basis presented in experiments made the lowest standard deviation at 50.000 simulated paths. This happens for most of cases and throughout strikes when the very same case is considered.

When analyzing one of the cases in study throughout strike prices, we observe that a specific basis delivers the lowest standard deviation in most of experiments. For example, in case 1, if it is a put option, then Hermite A delivers a price whose standard deviation is the lowest in two of the three set of parameters used. Whereas, for example, in case 3 for a call option across all strike prices considered, the polynomial basis that delivers a price with the lowest standard deviation is power. By repeating this process of column analysis of Table 8 , in most of strikes considered, when pricing an American Asian put option, Hermite A provides a better performance to the algorithm. On the other hand, when pricing an American Asian call option, Power provides a better performance through different strikes.

Considering a specific strike price throughout cases in Table 8, we figure out that the same feature happens, i.e., there is, in most cases, one polynomial basis whose price delivered has the lowest standard deviation. For example, for a put option whose strike price is 100 in three out of the four cases studied, Hermite A delivers the lowest standard deviation for the price estimates. Whereas, for a call option with the same strike price, the polynomial basis power provides a better performance, i.e., the lowest standard deviation in most cases. By proceeding this process of row analysis in Table 8, we got in most cases when pricing an American Asian put option that Hermite A had the best performance. Whereas when pricing an American Asian call option, Power delivers a better performance in most of cases.

These results suggest that pricing American Asian options using the Least-Squares Monte Carlo Method enables the selection of one polynomial basis, regardless of the kind of Amerasian. In fact, the main concern is whether we are pricing put or call option. 


\section{Conclusions}

Our experiments using the four chosen polynomial basis ratified one of the method's elements, i.e., that any orthogonal polynomial basis can be used for pricing American Asian options, since in all of the simulated paths the estimated prices of the options are virtually the same.

For all of the Amerasian options priced, and for each of the basis used, the convergence of the price estimate results from the decrease of the standard deviation while the number of simulated paths increases.

Considering 50,000 simulated trajectories, our study suggests that it is possible to empirically choose one specific polynomial basis for pricing an American Asian option. The results show that one polynomial basis is marginally more accurate than others, by providing a lower standard deviation it delivers a better performance to the algorithm when pricing a complex option as American Asian options. Such empirical outcome is theoretically unpredictable, since in principle all basis can be indistinctly used when pricing the derivative.

Therefore, for practical purposes, if a trader is looking for a faster and more accurate way to valuing an American Asian call option, the polynomial basis suggested to perform this procedure is Hermite A. On the other hand, if their concern is valuing an Amerasian put option, the Power polynomial basis is recommended. This speediness means being capable of measuring the worthiness of any derivative in order to screen accurately business opportunities not only for hedge purposes but also for arbitrage purposes.

\section{Endnotes}

${ }^{1}$ Where $\Omega$ is the set of all possible paths; $\mathfrak{I}$ is the filter of events at a certain time; and $Q$ is the risk-neutral probability measure of the elements of $\mathfrak{I}$

${ }^{2}$ Hilbert space is a vector space with internal product and complete regarding the rule set for this internal product

${ }^{3}$ The Fixed-Start Time Window Asian options are options whose number of elements' average increases in time, having its beginning at some point of the timeline

${ }^{4}$ The routines were programmed and performed in MATLAB 6.5.

Competing interests

The authors declare that they have no competing interests.

Authors' contributions

This article is part of Ms. USMdL dissetation of Master's degree got in Pontifical Catholic University of Rio de Janeiro, whose advisor was Professor CPS Ph.D. Both authors read and approved the final manuscript.

\footnotetext{
Authors' information

Ursula Silveira Monteiro de Lima holds a master's degree in Industrial Engeneering at Pontifical Catholic University of Rio de Janeiro - DEI/PUC-Rio - research field: Finance and Investment Analysis. Besides, Ms. Lima holds a B.A. in Economics at Getulio Vargas Foundation - FGV/EPGE and a Graduate Specialization in Finance and the Capital Markets at Getulio Vargas Foundation of São Paulo - FGV-SP.

Carlos Patricio Samanez is graduated in Industrial Engineering, M.Sc. in Production Engineering and Ph.D. in Financial Economics. Associate professor at Faculty of Economics - State University of Rio de Janeiro - UERJ, and Department of Industrial Engineering - Pontifical Catholic University of Rio de Janeiro - PUC-Rio.
}

Received: 7 February 2015 Accepted: 21 December 2015

Published online: 13 January 2016

References

Abramowitz M, Stegun IA (eds) (1972) Handbook of mathematical functions: with formulas, graphs, and mathematical tables. Dover Publications, New York 
Cerrato M, Cheung KK (2007) Valuing American style derivatives by least squares methods. Cent Int Capital Markets Discuss Papers 13:1-24

Chaudhary SK (2005) American options and the LSM algorithm: Quasi-random sequences and brownian bridges. J Comput Finance 8:4

Clement E, Lamberton D, Protter P (2002) An analysis of the Longstaff- Schwartz algorithm for american option pricing. Finance Stochast 6(4):449-471

Glasserman P (2004) Monte Carlo methods in financial engineering. Springer, New York

Lima EL (2007) Espaços métricos, 4th edn. Pure and Applied Mathematic Institute - IMPA, Rio de Janeiro

Longstaff FA, Schwartz ES (2001) Valuing american options by simulation: a simple least-squares approach. Rev Financ Stud 14(1):113-147

McDonald RL (2006) Derivative markets, 2nd edn. Addison Wesley, Boston

Moreno M, Navas JF (2003) On the robustness of Least-Squares Monte Carlo (LSM) for pricing american derivatives. Rev Deriv Res 6(2):107-128

Stentoft L (2004) Convergence of the least squares monte carlo approach to american option valuation. Manag Sci 50(9):1193-1203

Submit your manuscript to a SpringerOpen ${ }^{\circ}$ journal and benefit from:

- Convenient online submission

- Rigorous peer review

- Immediate publication on acceptance

Open access: articles freely available online

- High visibility within the field

- Retaining the copyright to your article

Submit your next manuscript at $>$ springeropen.com 